\title{
Cuestionario para la evaluación del optimismo: Fiabilidad y evidencias de validez
}

\section{Questionnaire for the assessment of optimism: Reliability and evidence of validity}

\author{
Ignacio Pedrosa \\ Universidad de Oviedo, España \\ Karem Celis-Atenas \\ Universidad de Santiago de Chile (USACH), Chile \\ Javier Suárez-Álvarez \\ Universidad de Oviedo, España \\ Eduardo García-Cueto \\ Universidad de Oviedo, España \\ José Muñiz \\ Universidad de Oviedo, España \\ ${ }^{\mathrm{c}}$ Centro de Investigación en Red de Salud Mental (CIBERSAM), España
}

(Rec: 2 octubre 2014 / Acept: 2 junio 2015)

\begin{abstract}
Resumen
El optimismo ha mostrado numerosos beneficios físicos y emocionales en diversas poblaciones y contextos profesionales. Sin embargo, los instrumentos para evaluarlo son escasos y presentan importantes limitaciones, especialmente en población joven. En el presente trabajo se presenta un cuestionario que, mediante 9 ítems, evalúa el optimismo disposicional y se analiza su relación con la personalidad e inteligencia emocional. La muestra estuvo compuesta por 2.693 jóvenes españoles $(M=16.52, D T=1.38)$, de los cuales el $51.10 \%$ fueron hombres. La inteligencia emocional se evaluó con el cuestionario Trait Meta-Mood Scale-24, mientras que los cinco grandes rasgos de personalidad se midieron mediante la escala Overall Personality Assessment Scale. El cuestionario de optimismo muestra una fiabilidad elevada $(\alpha=.84)$, siendo preciso para un amplio rango de habilidad $(\theta$ entre $-3 y+2)$, así como un ajuste aceptable a una estructura unidimensional. Se confirma la elevada relación entre el optimismo y los rasgos de personalidad e inteligencia emocional.

Palabras clave: evaluación, optimismo, personalidad, inteligencia emocional.
\end{abstract}

\begin{abstract}
Optimism has been shown to have numerous physical and emotional benefits in a range of populations and professional contexts. Nonetheless, there are few instruments for its evaluation and they have significant limitations, especially in young people. This work presents a questionnaire which, through 9 items, evaluates dispositional optimism and analyses its relationship to personality and emotional intelligence. The sample was made up of 2,963 Spanish adolescents of whom 51.10\% were male. Emotional intelligence was measured using the Trait Meta-Mood Scale-24, while the big-five personality traits were measured using the Overall Personality Assessment Scale. The optimism questionnaire demonstrated high reliability $(\alpha=.84)$, it was accurate over a wide ability range ( $\theta$ from -3 to +2 ) and gave an acceptable fit to a unidimensional structure. The clear relationship between optimism, personality traits and emotional intelligence was confirmed. Key words: assessment, optimism, personality, emotional intelligence.
\end{abstract}

\footnotetext{
Correspondencia:

Ignacio Pedrosa. Email: npedrosa@cop.es, Facultad de Psicología, Universidad de Oviedo. Pz/ Feijóo, s/n. 33003. Oviedo (España).

Agradecimientos:

Este trabajo ha sido parcialmente financiado por el Ministerio de Educación, Cultura y Deporte del Gobierno de España mediante sus programas FPU y FPI, por el Ministerio de Ciencia e Innovación, por los programas FONDECYT y por el Programa Formación de Capital Humano Avanzado de Chile (CONICYT). Referencias: AP2010-1999, PSI2011-28638, 21130624 y 1110520.
} 


\section{Introducción}

La psicología ha centrado tradicionalmente sus esfuerzos en resolver los múltiples problemas y trastornos del individuo bajo una visión negativa y patológica, hecho que, indiscutiblemente, ha generado numerosos avances en cuanto a investigación e intervención se refiere (PérezÁlvarez, 2013). Sin embargo, en las últimas décadas ha surgido una alternativa en la intervención psicopatológica que propone afrontar estas situaciones desde un enfoque positivo. Esta orientación ha demostrado el importante efecto potencial que variables como la felicidad, el afecto positivo o el optimismo, entre otras, presentan respecto al bienestar físico y emocional (Gander, Proyer, Ruch y Wyss, 2013; Park, Peterson y Sun, 2013; Schmitt, Gielnik, Zacher y Klemann, 2013; Seligman, 2008; Vera-Villarroel, Pavez y Silva, 2012).

El optimismo destaca como una de las variables más representativas en el ámbito de la psicología positiva. Para su definición cabe destacar tres enfoques: $a$ ) basado en una perspectiva disposicional, b) como sinónimo de esperanza o c) como un estilo explicativo (Peterson, 2000; Rauch, Schweizer y Moosbrugger, 2008). El concepto de esperanza presenta matices diferenciadores respecto al optimismo, al definirse por objetivos concretos que la persona debe alcanzar tras el análisis de su situación (Snyder, Rand y Sigmon, 2005). El estilo explicativo implica una atribución causal, interna o externa, de los diferentes eventos negativos sufridos por la personas de manera cotidiana (Peterson y Seligman, 1984). Por su parte, el optimismo disposicional se define como una tendencia personal a evaluar de forma positiva la ocurrencia de eventos futuros (posibles o no), siendo una característica estable temporal y situacionalmente, lo que genera una tendencia a persistir en la tarea cuando ésta se dirige hacia una meta (Carver, Scheier y Segerstrom, 2010; Rauch, Schweizer y Moosbrugger, 2007; Scheier y Carver, 1985). Teniendo en cuenta estas tres perspectivas, se considera la disposicional como la más adecuada para definir y evaluar este constructo.

El optimismo ha mostrado un efecto positivo en diferentes contextos como el clínico, el económico o el educativo, constituyendo una variable relevante a la hora de diseñar intervenciones en cualquiera de estos ámbitos (Londoño, 2009; López Puga y García García, 2011; Xanthopoulou, Bakker y Fischbach, 2013). Además, se ha observado su elevada relación con diferentes rasgos de personalidad y la inteligencia emocional. Las personas optimistas muestran menores niveles de neuroticismo y mayores niveles de extraversión y estabilidad emocional, así como una relación positiva con la amabilidad y responsabilidad (Carver y Connor-Smith, 2010; Carver et al., 2010; Kam y Meyer, 2012). Por otra parte, aquellos trabajos que han analizado su relación con la inteligencia emocional percibida, han puesto de manifiesto correlaciones positivas del optimismo con la claridad y reparación emocional, siendo ésta negativa en el caso de la atención emocional (Augusto-Landa, PulidoMartos y López-Zafra, 2011; Augusto Landa, Aguilar Luzón y Salguero de Ugarte, 2008; Extremera, Durán y Rey, 2007).

En cuanto a la evaluación del constructo, existen diversos instrumentos destinados a tal fin, entre los que destacan el cuestionario de optimismo social (Schweizer y Koch, 2001), el cuestionario de optimismo y pesimismo de Seligman (2003), el cuestionario de orientación vital (LOT; Scheier y Carver, 1985) y su versión revisada (LOT-R; Scheier, Carver y Bridges, 1994), siendo estos dos últimos los más utilizados internacionalmente. El cuestionario LOT está formado por diez ítems (tres evalúan optimismo, tres pesimismo y los cuatro ítems restantes presentan una finalidad distractora que evitan hacer tan evidente el contenido del test). Ha sido traducido y adaptado a múltiples idiomas como español, francés o griego, entre otros (Ferrando, Chico y Tous, 2002; Laranjeira, 2008; Lyrakos, Damigos, Mavreas, Georgia y Dimoliatis, 2010). Sin embargo, aun siendo una escala frecuentemente utilizada, tanto sus propiedades psicométricas como las diferentes limitaciones que presenta parecen mostrar que puede suponer una escala poco adecuada para evaluar este constructo. Así, la fiabilidad del LOT-R, estimada mediante el coeficiente $\alpha$ de Cronbach, ha mostrado valores muy dispares, y en algunos casos excesivamente reducidos, tanto para la escala completa (entre .58 y .83), como para las dimensiones de optimismo (entre .65 y .75 ) y pesimismo (entre .45 y .79). Estos resultados son aun menos alentadores cuando la escala se aplica sobre población adolescente, mostrando coeficientes $\alpha$ entre .51 y .63 (Glaesmer et al., 2012; Jovanovic y Gavrilov-Jerkovic, 2013; Vera-Villarroel, Córdova-Rubio y Celis-Atenas, 2009a, 2009b), una fiabilidad inferior a la hallada en el trabajo de Monzani, Steca y Greco (2014), la cual fue de $\Omega=.76$. Sin embargo, hay que destacar que este último se basa en una perspectiva unidimensional, la cual difiere de la bidimensionalidad del LOT-R propuesta originalmente.

Además de la reducida fiabilidad mostrada por el instrumento, hay que añadir la recurrente inconsistencia en cuanto a su estructura factorial, siendo posible encontrar trabajos que abogan tanto por su unidimensionalidad como por la bidimensionalidad originalmente planteada por sus autores 
(Monzani et al., 2014; Rauch et al., 2007; Segerstrom, Evans y Eisenlohr-Moul, 2011; Zenger et al., 2013).

El LOT-R cuenta con otras importantes limitaciones tales como: a) su inadecuada validez de contenido en cuanto a representación del domino (Sireci y Faulkner-Bond, 2014), pues su excesivamente reducido número de ítems limita la posibilidad de evaluar la estabilidad de la visión de futuro ante diversas situaciones de la vida (Londoño, Hernández, Alejo y Pulido, 2013); b) los posibles errores de estimación de sus coeficientes de fiabilidad en determinadas muestras al haberse analizado sobre tamaños muestrales muy dispares siendo, en algunos casos, tamaños reducidos (Brown, 1999); c) las contradicciones mostradas por algunos indicadores del test en estudios transculturales, viéndose afectado por las propias diferencias culturales y la importante heterogeneidad de las muestras en que ha sido aplicado (Fischer y Chalmers, 2008; Jovanovic y Gavrilov-Jerkovic, 2013; Londoño et al., 2013); d) y la falta, hasta la fecha, de resultados derivados de los nuevos desarrollos psicométricos de la Teoría de Respuesta al Ítem.

Debido a este conjunto de limitaciones que el LOT-R presenta, Londoño et al. (2013) han tratado de proponer un nuevo instrumento de medida, aunque las propiedades psicométricas mostradas hasta la fecha son relativamente reducidas. Un ejemplo de ello es que el coeficiente de fiabilidad de esta nueva escala, estimado en una muestra de 431 jóvenes, fue de $\alpha=.73$ (Gómez-Acosta y Londoño, 2013).

La falta de tests adecuados para la medida de este constructo se agudiza cuando se pretende evaluar población joven, pues no existen instrumentos que hayan mostrado adecuadas propiedades psicométricas en esta población. Así, diferentes instrumentos específicamente diseñados para este grupo de edad, como el Test de Orientación Vital para jóvenes (YLOT; Ey et al., 2005), el Test de Optimismo-Pesimismo (OPTI; Stipek, Lamb y Zigler, 1981) o el Test de orientación vital en niños valorado por los padres (PLOT; Lemola et al., 2010), presentan inconvenientes en su aplicación, pues requieren altos niveles de introspección y razonamiento para poder responder a sus ítems o exigen una evaluación complementaria de los padres del participante.

Teniendo en cuenta lo expuesto previamente, puede decirse que no existe ningún instrumento de fácil aplicación que permita evaluar el optimismo en población juvenil de forma válida y fiable. Por todo ello, el objetivo central del presente trabajo es desarrollar un nuevo instrumento de medida para la evaluación del optimismo disposicional en población joven. Aparte de los enfoques psicométricos clásicos, se utilizarán los desarrollos recientes derivados de la TRI, los cuales permiten un análisis más preciso de las propiedades psicométricas de los instrumentos de medida (De Ayala, 2009; Hambleton, Swaminathan y Rogers, 1991). También se aportan evidencias de validez relativas a la integración del constructo optimismo dentro de la estructura de la personalidad y la inteligencia emocional percibida. Finalmente, se analizan las diferencias en optimismo en función del sexo y la edad.

\section{Método}

\section{Participantes}

La muestra estuvo formada por 2.693 participantes, los cuales pertenecían a 22 centros educativos de tres comunidades autónomas situadas en el Norte de España: Principado de Asturias, Cantabria y Castilla-León. De ellos, un 34.20\% cursaba estudios de $4^{\circ}$ de educación secundaria obligatoria, un $57.90 \%$ realizaba estudios de bachillerato y el $7.80 \%$ restante desarrollaba estudios de formación profesional.

La edad de los participantes estuvo comprendida entre los 14 y los 23 años, con una media de 16.52 y una desviación típica de 1.38. En cuanto al sexo, el $51.10 \%$ fueron hombres.

\section{Instrumentos}

Los rasgos de personalidad fueron evaluados mediante el Overall Personality Assessment Scale (OPERAS; Vigil-Colet, Morales-Vives, Camps, Tous y Lorenzo-Seva, 2013). Este instrumento evalúa los cinco grandes rasgos de la personalidad (extraversión, estabilidad emocional, responsabilidad, amabilidad y apertura a la experiencia) mediante 7 ítems por dimensión. Las subescalas presentan coeficientes de fiabilidad entre .71 y .86 (Vigil-Colet et al., 2013). Además, como señalan Vigil-Colet et al. (2013), el instrumento presenta evidencias adecuadas de validez convergente.

La inteligencia emocional percibida ha sido medida con la versión española de la escala Trait Meta-Mood Scale en su versión breve (TMMS-24; Fernández-Berrocal, Extremera y Ramos, 2004). El instrumento está constituido por las subescalas de atención, claridad y reparación emocional, las cuales presentan índices de fiabilidad entre .72 y .86 en población adolescente (Díaz Castela et al., 2013; Extremera et al., 2007; Salguero, Fernández-Berrocal, Balluerka y Aritzeta, 2010).

Para evaluar el optimismo se desarrolló el Cuestionario de Optimismo (COP), formada por 10 ítems con un formato 
de respuesta tipo Likert de 5 puntos, donde 1 significaba estar totalmente en desacuerdo y 5 totalmente de acuerdo. El uso de una escala de 5 puntos se debe a que es entre 4 y 6 categorías de respuesta cuando los parámetros psicométricos se estiman con mayor precisión (Lozano, García-Cueto y Muñiz, 2008). De manera complementaria se incluyó la pregunta "Soy una persona optimista", con el propósito de tener una estimación subjetiva global del optimismo de los participantes.

En cuanto a la redacción de los ítems del cuestionario COP, además de focalizar la atención en la validez de contenido de los mismos, se prestó especial atención a su redacción, para que fuesen perfectamente comprendidos por cualquier tipo de población y rango de edad, lo que permite generalizar la administración del instrumento a otras poblaciones y contextos.

El desarrollo de los ítems se realizó tras consultar los diferentes instrumentos existentes para la evaluación del optimismo y teniendo en cuenta las recomendaciones psicométricas internacionales (Downing, 2006).

\section{Procedimiento}

Las pruebas fueron aplicadas por psicólogos expertos en la administración de tests. La evaluación se realizó de manera colectiva en las aulas proporcionadas por cada centro educativo. La participación fue totalmente voluntaria, sin ninguna recompensa y bajo los requisitos exigidos por el Comité de Ética en Investigación de la Facultad de Psicología de la Universidad de Oviedo.

\section{Análisis de datos}

Para analizar las propiedades psicométricas del cuestionario de optimismo (COP), se llevó a cabo un análisis de los ítems a través de sus índices de discriminación.

Posteriormente, se evaluó el Funcionamiento Diferencial de los Ítems (DIF) por sexo mediante regresión logística (Zumbo, 1999). Además del test de significación estadística, se valoró el tamaño del efecto para reducir la tasa de posibles falsos positivos (Gómez-Benito, Hidalgo y Zumbo, 2013).

Para probar su estructura factorial, la muestra se dividió en tres submuestras aleatorias, sobre las que se aplicaron un análisis factorial exploratorio y dos análisis factoriales confirmatorios, respectivamente.

Sobre la primera submuestra $\left(\mathrm{n}_{1}\right)$ se exploró su estructura factorial, utilizando la matriz de correlaciones policóricas y el método de extracción de mínimos cuadrados no ponderados. El número de factores se determinó a partir del procedimiento de Análisis Paralelo (Timmerman y Lorenzo-Seva, 2011), bajo la aleatorización de 5,000 matrices de correlaciones.

Sobre las dos submuestras restantes $\left(\mathrm{n}_{2} \mathrm{y}_{3}\right)$ se aplicaron sendos análisis factoriales confirmatorios mediante el procedimiento de validación cruzada (Browne y Cudeck, 1993). Se empleó el método de estimación de mínimos cuadrados ponderados, debido al análisis de variables categóricas y al elevado tamaño muestral (Flora y Curran, 2004). El modelo propuesto en esta submuestra se mantuvo idéntico en la tercera submuestra, sobre la que se aplicó un nuevo análisis factorial confirmatorio. El ajuste de los datos al modelo se probó a partir del índice de ajuste comparativo (CFI), índice de Tucker-Lewis (TLI) y error medio cuadrático de aproximación (RMSEA). El peso factorial de cada ítem en la variable se estimó sobre el total de participantes.

La fiabilidad del cuestionario se estimó mediante el coeficiente $\alpha$ para datos ordinales (Elosua y Zumbo, 2008).

Dentro del marco de la Teoría de Respuesta al Ítem se estimaron los parámetros de dificultad y discriminación de los ítems del cuestionario de optimismo bajo el modelo graduado de Samejima (Samejima, 1969). Teniendo en cuenta los criterios propuestos por Baker (2001), valores de 0.01-0.24 para el parámetro $a$ se pueden considerar muy bajos, de 0.25-0.64 bajos, entre 0.65-1.34 moderados, con un rango de 1.35-1.69 altos y superiores a 1.7 muy altos. Se estimó la información aportada por cada uno de los ítems (FII) y por el test total (FIT) para niveles de habilidad en un rango de $\theta= \pm 3$ en pasos de un punto.

Para el estudio de diferencias en función del sexo y las diferentes edades de los participantes, se emplearon las pruebas U de Mann-Whitney y Kruskal-Wallis, respectivamente $(\mathrm{NC}=95 \%)$, debido a la no-normalidad de los datos, probada con la prueba de Shapiro-Wilk. Además, se calculó el tamaño del efecto empleando los métodos Delta de Cliff (1993) y $V$ de Cramér (1946), recomendados ambos cuando se violan los supuestos de normalidad y se emplean escalas en formato Likert (Macbeth, Razumiejczyk y Daniel Ledesma, 2011).

Se elaboró el baremo del COP en una escala de decatipos.

Con objeto de evaluar la relación entre el optimismo, los rasgos de personalidad y la inteligencia emocional, se calcularon el coeficiente de correlación de Pearson, el coeficiente de determinación y el coeficiente beta entre el optimismo y cada dimensión de los rasgos de personalidad (OPERAS) y la inteligencia emocional (TMMS-24). 


\section{Resultados}

\section{Análisis de los items del cuestionario de optimismo}

Mediante el estudio de los índices de discriminación de los diez ítems que inicialmente componían el cuestionario de optimismo, se eliminó uno de ellos por presentar un valor inferior a .30 (Muñiz, Fidalgo, García-Cueto, Martínez y Moreno, 2005). Los índices de discriminación de los nueve ítems restantes fueron elevados en todos los casos, situándose en un rango entre .38 y .61 (Tabla 1).

En cuanto al DIF, ninguno de estos nueve ítems resultó estadísticamente significativo, siendo, además, el tamaño del efecto prácticamente nulo en todos ellos (Gómez-Benito et al., 2013), descartando así la existencia de DIF por sexo.

Teniendo en cuenta los resultados en ambos análisis, el cuestionario de optimismo quedó formada por 9 ítems.

\section{Evidencias de validez factorial del cuestionario de optimismo}

En primer lugar, se exploró la estructura factorial del cuestionario de optimismo aplicando un análisis factorial exploratorio sobre la primera submuestra $\left(\mathrm{n}_{1}=873\right)$. Los datos confirmaron la adecuación muestral para la aplicación del análisis factorial (Coeficiente Kaiser-Meyer-Olkin, $\mathrm{KMO}=.86$; Estadístico de Bartlett, $p<.001)$. Además, a partir de los índices de ajuste, se puede definir un único factor estadísticamente significativo (índice de bondad del ajuste global, $\mathrm{GFI}=.98$; raíz cuadrática media de los residuales, RMSR $=.069$ ), el cual explica el $42.43 \%$ de la varianza total.

Sobre las dos submuestras restantes se aplicaron dos análisis factoriales confirmatorios que permitieran probar la estructura unidimensional anteriormente definida. Como se puede ver en la Tabla 2, todos ellos se encuentran en torno a valores adecuados.

Tabla 1. Índices de discriminación y pesos factoriales del cuestionario de optimismo (COP).

\begin{tabular}{llll}
\hline & \multicolumn{1}{c}{ Ítem } & Índice de discriminación & Peso factorial \\
\hline 1. & Creo que conseguiré las principales metas de mi vida & .49 & .64 \\
2. & Cuando pienso en el futuro soy positivo & .57 & .80 \\
3. & Me ocurren más cosas buenas que malas & .42 & .54 \\
4. & Pienso que todo saldrá mal & .55 & .71 \\
5. & Veo cada reto como una oportunidad de éxito & .38 & .52 \\
6. & Por muy mal que salgan las cosas encuentro aspectos positivos & .54 & .78 \\
7. & Veo los aspectos positivos de las cosas & .61 & .87 \\
8. & Tengo confianza en superar los problemas & .55 & .68 \\
9. & Tengo confianza en el futuro & .59 & .77 \\
\hline
\end{tabular}

Nota: El ítem 4 debe ser redireccionado para obtener la puntuación total en el cuestionario.

Tabla 2. Índices de ajuste de los análisis factoriales confirmatorios sobre las submuestras $n_{2 y} n_{3}$.

\begin{tabular}{cccc}
\hline Submuestra & CFI & TLI & RMSEA \\
\hline $\mathrm{n}_{2}=901$ & .89 & .95 & 0.113 \\
$\mathrm{n}_{3}=919$ & .90 & .87 & 0.109 \\
\hline
\end{tabular}

Nota: $C F I=I ́ n d i c e$ de ajuste comparativo; TLI=Índice de Tucker-Lewis; RMSEA=error medio cuadrático de aproximación. 
Además, se estimó el peso factorial de cada ítem en el factor (Tabla 1), siendo todos ellos estadísticamente significativos $(\mathrm{p}<.001)$.

Finalmente, sobre el cuestionario COP se añadieron evidencias de la estimación global del optimismo por parte de los participantes mediante la correlación entre la puntuación total en el instrumento y la respuesta a la pregunta destinada a tal fin. La correlación obtenida fue de $r_{\mathrm{xy}}=.64$, lo que demuestra una convergencia adecuada con la medida del constructo.

\section{Fiabilidad de los instrumentos de medida}

La fiabilidad del cuestionario de optimismo desarrollado mostró un coeficiente $\alpha$ para datos ordinales de .84. Por su parte, tanto las dimensiones del TMMS-24 como del OPERAS presentaron coeficientes de fiabilidad adecuados en esta muestra (Tabla 3).

\section{Análisis del cuestionario COP mediante modelos de} Teoría de Respuesta al Ítem

Se estimaron los parámetros de dificultad y discriminación para cada uno de los ítems del cuestionario (Tabla 4). La mayoría de ítems presentan un poder discriminativo alto y muy alto. En cuanto a su dificultad, aquellos ítems que se focalizan directamente en retos o problemas de manera específica (ítems 1,5 y 8) son los que muestran parámetros $b_{l}$ más bajos, así como una mayor distancia respecto al nivel de dificultad de cada una de las alternativas de respuesta restantes $\left(b_{2}, b_{3} y b_{4}\right)$.

La Tabla 5 muestra la información aportada por cada ítem y por el test total para niveles de habilidad entre \pm 3 . La mayor parte de ítems, y en consecuencia del test, muestran una información muy elevada hasta niveles de $\theta=+2$. Únicamente los ítems $1,3,5$ y 6 aportan un nivel

Tabla 3. Consistencia interna de las subescalas del Trait Meta-Mood Scale-24 y el Overall Personality Assessment Scale.

\begin{tabular}{|c|c|c|}
\hline & Dimensiones & $\alpha$ \\
\hline \multirow{3}{*}{ IE } & Atención emocional & .89 \\
\hline & Claridad emocional & .83 \\
\hline & Reparación emocional & .78 \\
\hline \multirow{5}{*}{$\mathrm{RP}$} & Amabilidad & .71 \\
\hline & Apertura a la experiencia & .75 \\
\hline & Estabilidad emocional & .81 \\
\hline & Extraversión & .80 \\
\hline & Responsabilidad & .71 \\
\hline
\end{tabular}

Nota: IE=Inteligencia Emocional; $R P=$ Rasgos de Personalidad.

Tabla 4. Parámetros del cuestionario de optimismo (COP) estimados bajo el Modelo Graduado de Samejima.

\begin{tabular}{cccccc}
\hline Ítems & $\mathrm{a}$ & $\mathrm{b}_{1}$ & $\mathrm{~b}_{2}$ & $\mathrm{~b}_{3}$ & $\mathrm{~b}_{4}$ \\
\hline 1 & 1.28 & -3.89 & -2.44 & -0.60 & 1.25 \\
2 & 1.69 & -2.69 & -1.69 & -0.60 & 0.67 \\
3 & 1.04 & -3.28 & -1.98 & -0.12 & 1.54 \\
4 & 1.62 & -2.85 & -2.03 & -1.02 & 0.02 \\
5 & 0.98 & -4.93 & -2.79 & -0.55 & 1.55 \\
6 & 1.54 & -2.86 & -1.42 & -0.13 & 1.38 \\
7 & 1.88 & -2.82 & -1.72 & -0.41 & 1.00 \\
9 & 1.60 & -3.49 & -2.06 & -0.60 & -1.15 \\
\hline
\end{tabular}


Tabla 5. Información aportada por los ítems y el cuestionario de optimismo (COP), en función de los diferentes niveles de habilidad.

\begin{tabular}{cccccccc}
\hline \multirow{2}{*}{ Ítems } & \multicolumn{7}{c}{ Nivel de habilidad } \\
\cline { 2 - 8 } & -3.0 & -2.0 & -1.0 & 0.0 & 1.0 & 2.0 & 3.0 \\
\hline 1 & 0.36 & 0.34 & 0.34 & 0.31 & 0.36 & 0.21 & 0.04 \\
2 & 0.34 & 0.42 & 0.41 & 0.38 & 0.33 & 0.08 & 0.01 \\
3 & 0.39 & 0.39 & 0.28 & 0.38 & 0.34 & 0.29 & 0.07 \\
4 & 0.37 & 0.43 & 0.42 & 0.39 & 0.15 & 0.02 & 0.00 \\
5 & 0.36 & 0.25 & 0.31 & 0.30 & 0.30 & 0.29 & 0.07 \\
6 & 0.36 & 0.36 & 0.39 & 0.39 & 0.36 & 0.25 & 0.05 \\
7 & 0.36 & 0.41 & 0.38 & 0.37 & 0.38 & 0.15 & 0.02 \\
8 & 0.36 & 0.39 & 0.37 & 0.32 & 0.37 & 0.18 & 0.03 \\
9 & 0.34 & 0.41 & 0.41 & 0.37 & 0.37 & 0.14 & 0.02 \\
Test & 3.24 & 3.40 & 3.31 & 3.21 & 2.96 & 1.61 & 0.31 \\
\hline
\end{tabular}

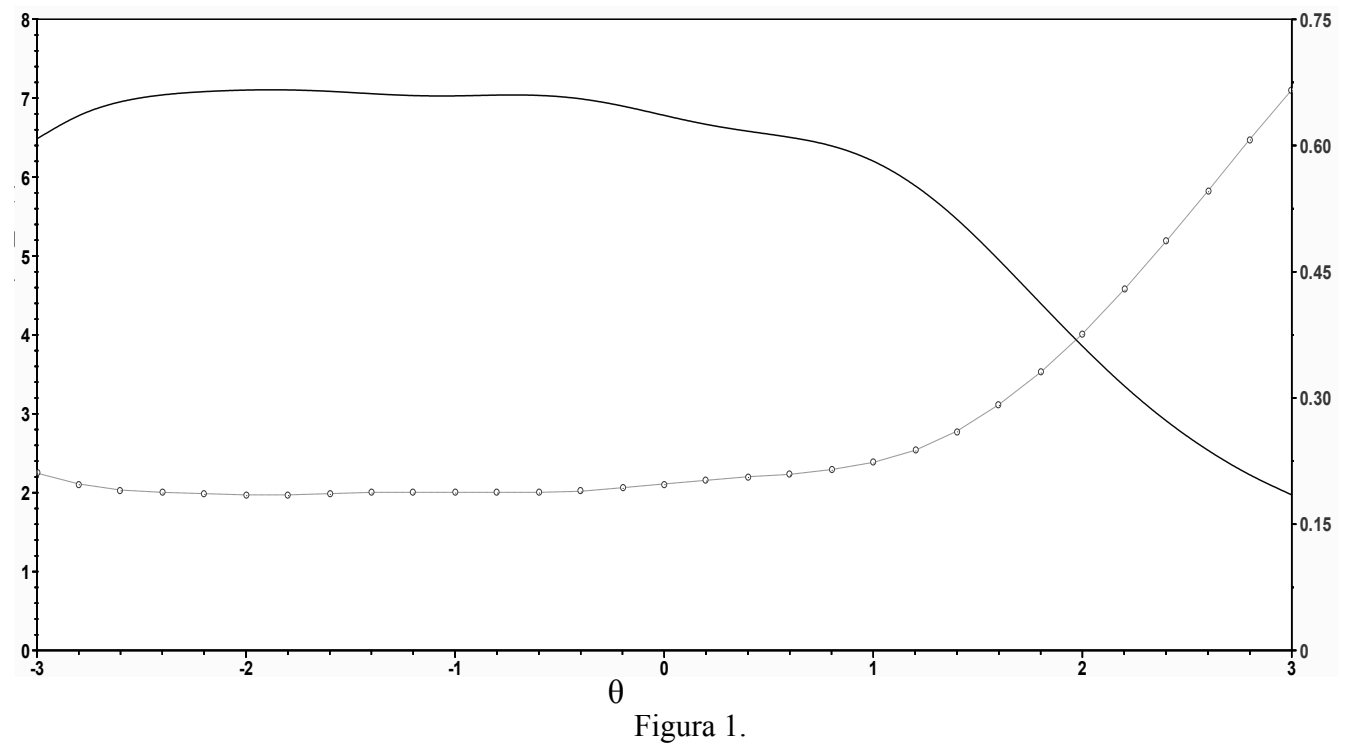

Función de información del cuestionario de optimismo.

de información elevado para aquellos participantes con un nivel de optimismo en torno a 2.

Para analizar la precisión de medida aportada por el COP, se estimó su función de información bajo el modelo graduado de Samejima (Figura 1). Acorde a la FIT mostrada en la Tabla 5, la Función de Información muestra una elevada precisión para los niveles bajos y medios de optimismo, disminuyendo su precisión a partir de estimaciones de $\theta$ superiores a +2 . Los valores mostrados en la Figura 1 son una transformación lineal de la información aportada por cada ítem y por el test total de la Tabla 5.
Diferencias en optimismo en función del sexo y la edad

La prueba de Kruskal-Wallis mostró la inexistencia de diferencias estadísticamente significativas en función de la edad de los participantes $(p=.70)$, siendo además el tamaño del efecto obtenido prácticamente nulo $\left(\varphi_{\mathrm{c}}=.02\right)$.

La prueba U de Mann-Whitney, por su parte, sí detectó diferencias estadísticamente significativas en función del sexo $(p<.001)$, mostrando los hombres un mayor optimismo. Sin embargo, el tamaño del efecto fue reducido $(\delta=.11)$. 
Tabla 6. Baremo del cuestionario de optimismo

\begin{tabular}{cc}
\hline PD & Decatipo \\
\hline $9-23$ & 0 \\
$24-25$ & 1 \\
$26-28$ & 2 \\
$29-30$ & 3 \\
$31-32$ & 4 \\
$33-35$ & 5 \\
$36-37$ & 6 \\
$38-39$ & 7 \\
$40-41$ & 8 \\
$42-44$ & 9 \\
45 & 10 \\
\hline
\end{tabular}

Nota: $P D=$ Puntuación Directa.

Tabla 7. Coeficientes de correlación y regresión múltiple entre optimismo, rasgos de personalidad e inteligencia emocional.

\begin{tabular}{lcccc}
\hline & & Optimismo & $\mathrm{R}^{2}$ & Beta \\
\hline \multirow{3}{*}{ Inteligencia emocional } & Atención emocional & -.06 & & -.19 \\
& Claridad emocional & .34 & .44 & .22 \\
& Reparación emocional & .62 & .56 \\
& Amabilidad & .26 & .08 \\
Rasgos de personalidad & Apertura & .15 & .10 \\
& Estabilidad emocional & .62 & .47 & .52 \\
& Extraversión & .31 & .18 \\
\hline
\end{tabular}

\section{Baremo del cuestionario de optimismo}

A partir de estos resultados se aporta el baremo del instrumento desarrollado para la interpretación de sus puntuaciones en una escala de decatipos (Tabla 6).

\section{Optimismo, personalidad e inteligencia emocional}

Finalmente, con objeto de integrar el constructo optimismo dentro de la estructura de la personalidad y la inteligencia emocional percibida en población juvenil, se calculó el coeficiente de correlación de Pearson $\left(r_{x y}\right)$, el coeficiente de determinación y el coeficiente beta entre las dimensiones que definen estos tres constructos (Tabla 7). Como se observa, la varianza común entre el optimismo y las dimensiones de inteligencia emocional fue de .44 , obteniendo un valor similar $\left(\mathrm{R}^{2}=.47\right)$ en el caso de los rasgos de personalidad.

\section{Discusión y conclusiones}

El optimismo ha demostrado numerosos beneficios respecto al bienestar físico y psicológico de las personas, tanto en población clínica como general, así como en diferentes contextos culturales y profesionales (Carver et al., 2010; Seligman, 2008; Vera-Villarroel et al., 2012). A pesar de la 
relevancia del optimismo en el ámbito psicológico, los instrumentos existentes para su evaluación presentan importantes limitaciones en cuanto a sus propiedades psicométricas, procedimientos de aplicación y, en muchos casos, siendo sólo posible emplearlos en contextos específicos (Fischer y Chalmers, 2008; Londoño et al., 2013). Por este motivo, se presenta un nuevo instrumento de medida (COP) que permite evaluar esta variable de forma breve, válida y fiable con independencia del contexto de aplicación y de la edad de la población diana.

El análisis de los ítems del cuestionario COP muestra cómo, tras eliminar uno de los ítems iniciales, todos los ítems restantes desarrollados para la evaluación del constructo presentan índices de discriminación elevados (superiores a .38), sin mostrar ninguno de ellos un funcionamiento diferencial por sexo. Estos datos implican que el instrumento final quede constituido por 9 ítems.

En cuanto a las evidencias de validez factorial del cuestionario, a partir de los adecuados resultados del análisis factorial exploratorio, parece posible definir el constructo optimismo como una variable unidimensional, la cual explica el $44.32 \%$ de la varianza. Esta hipótesis de unidimensionalidad se confirma a partir de los índices de ajuste obtenidos en los análisis factoriales confirmatorios. Así, aunque el ajuste absoluto (RMSEA) excede ligeramente los valores que serían deseables idealmente (i.e. 0.1; Browne y Cudeck, 1993), las medidas de ajuste incremental (CFI y TLI) asumen valores razonables en ambas submuestras (Kline, 2011).

Estos resultados concuerdan con trabajos precedentes en donde se sostiene la unidimensionalidad del constructo (Bandeira, Bekou, Silva Lott, Teixeira y Silva Rocha, 2002; Laranjeira, 2008), frente a otros trabajos que abogan por su bidimensionalidad, tanto en población general (Rauch et al., 2007; Segerstrom et al., 2011) como juvenil (Nakano, 2004; Sulkers et al., 2013). Cabe destacar cómo todos estos trabajos en que se defiende la estructura bidimensional del optimismo disposicional, han empleado el LOT como instrumento de evaluación. Los resultados parecen confirmar la hipótesis propuesta por Monzani et al. (2014), quienes, tras comparar diferentes estructuras factoriales en una muestra de 698 adolescentes, sostienen su unidimensionalidad. Así, estos autores confirman cómo la bidimensionalidad originalmente propuesta en el LOT-R es un resultado artificial debido a la redacción en forma positiva y negativa de los ítems y no a una estructura bifactorial realmente subyacente, la cual no refleja la naturaleza teórica real del constructo.

Todas las cargas factoriales de los ítems fueron superiores a .52 , lo que da cuenta de la alta relevancia que todos los ítems presentan en el constructo. La correlación hallada entre la puntuación total en el cuestionario COP y la pregunta "Soy una persona optimista" $\left(r_{\mathrm{xy}}=.64\right)$, por lo que parece sensato pensar en una adecuada evaluación del constructo.

Respecto a la consistencia interna del cuestionario de optimismo, se puede considerar que, a pesar de la relativa brevedad del mismo (9 ítems), su fiabilidad es adecuada $(\alpha=.84)$ en comparación con las escalas desarrolladas hasta la fecha (Glaesmer et al., 2012; Jovanovic y Gavrilov-Jerkovic, 2013; Londoño et al., 2013).

La mayor parte de los ítems presentan un parámetro $a$ alto-muy alto, mostrando parámetros $b$ con mayor distancia entre ellos en aquellos casos en los que el ítem se centra en problemas o retos que la persona debe asumir, lo que implica un pensamiento más resistente al cambio en estos casos. Por otro lado, tanto las FII como la FIT muestran una elevada precisión en la medida hasta niveles muy elevados de optimismo $(\theta=+2)$. Si bien el cuestionario es preciso para un rango muy amplio de participantes, resulta menos recomendable cuando se pretende evaluar a participantes con niveles de optimismo excesivamente elevados.

De cara a la aplicación de la COP, se aporta el baremo del instrumento en decatipos. Como se ha podido comprobar, existen diferencias estadísticamente significativas en función del sexo, siendo los hombres quienes tienen una visión más optimista del mundo que les rodea. Sin embargo, el tamaño del efecto obtenido es muy reducido $(\delta=.11$; Macbeth et al., 2011), lo que podría indicar que realmente las diferencias se deben al amplio tamaño muestral y no a diferencias inherentes a la propia variable en ambos sexos. Como muestran trabajos precedentes, existe una cierta inconsistencia en cuanto a las diferencias en función del sexo (Eloranta et al., 2012; Plagnol y Easterlin, 2008; Puskar et al., 2010). En opinión de autores como Tusaie y Patterson (2006), aunque en general los adolescentes no perciben la posibilidad de sufrir eventos negativos, parece que las mujeres se consideran más vulnerables ante los diferentes eventos estresantes, causando esto un nivel de optimismo ligeramente inferior respecto a los hombres.

En el caso de la edad, si bien no hay diferencias estadísticamente significativas, los participantes más jóvenes presentan la puntuación más baja en el cuestionario, mientras que son los de mayor edad quienes muestran el mayor nivel de optimismo. Diferentes autores han mostrado cómo el inicio de la etapa adolescente coincide con importantes cambios evolutivos asociados, a su vez, a niveles reducidos de optimismo, los cuales se incrementan progresivamente a medida que el adolescente se desarrolla (Pasch, Nelson, Lytle, Moe y Perry, 2008). 
En cuanto a la relación del optimismo con otras variables, dentro de los rasgos de personalidad, las mayores correlaciones se observan entre estabilidad emocional $\left(r_{x y}=.62\right)$ y responsabilidad $\left(r_{x y}=.33\right)$, siendo éstas igualmente elevadas y positivas respecto a las dimensiones de amabilidad y extraversión (Kam y Meyer, 2012; Sharpe, Martin y Roth, 2011). Sin embargo, cuando se analiza en qué medida cada uno de estos rasgos influye sobre la puntuación en optimismo, se observa cómo mientras la estabilidad emocional se considera una variable relevante, la amabilidad constituye la variable con menor repercusión a la hora de determinar las puntuaciones en optimismo. Las correlaciones respecto a la inteligencia emocional siguen, igualmente, el patrón de trabajos precedentes. Así, la correlación entre el optimismo y la atención sobre los sentimientos es negativa, aunque prácticamente nula, mientras que ésta es positiva en relación a la capacidad para entender las emociones propias y ajenas y la capacidad para regular los estados emocionales, siendo especialmente elevada en este último caso $\left(r_{x y}=.62\right.$; Augusto-Landa et al., 2011; Extremera et al., 2007). Acerca del efecto que cada una de las dimensiones ejerce sobre el optimismo, éste se puede considerar acorde a las correlaciones obtenidas: la reparación emocional presenta el mayor coeficiente de regresión, mientras que la atención emocional muestra un coeficiente negativo. En definitiva, las relaciones encontradas entre los diferentes rasgos de personalidad y las dimensiones de la inteligencia emocional percibida confirman la validez en relación a otras variables del instrumento (Augusto-Landa et al., 2011; Carver et al., 2010), y el grado en que éstas determinan las puntuaciones en optimismo se puede considerar moderado.

Considerando los resultados precedentes, puede concluirse que la COP permite evaluar el optimismo de forma breve, válida y fiable encontrándose, además, libre de cualquier contaminación contextual, lo que permite su aplicación en todo tipo de ámbitos y rango de edad. En cuanto a su estructura factorial, los resultados confirman la hipótesis de unidimensionalidad del constructo en población adolescente, en contraste con la propuesta bidimensional aportada por aquellos autores que, generalmente, emplean el LOT como instrumento de medida (Monzani et al., 2014).

De cara a futuras investigaciones, se considera necesario analizar las propiedades psicométricas del presente cuestionario en población adulta para completar su validación en población general. El desarrollo de un estudio transcultural permitiría probar la estabilidad del constructo, así como analizar el efecto que diversas variables socioculturales pueden ejercer sobre el optimismo.
Como principales limitaciones del estudio pueden considerarse tanto el uso exclusivo de datos de auto-informe para la evaluación de los diferentes constructos, con los posibles sesgos y limitaciones que ello conlleva, como la falta de un criterio externo que permita estudiar la validez convergente del cuestionario COP aquí desarrollado.

\section{Referencias}

Augusto-Landa, J. M., Pulido-Martos, M. y López-Zafra, E. (2011). Does Perceived Emotional Intelligence and Optimism/pessimism Predict Psychological Well-being? Journal of Happiness Studies, 12, 463-474. doi:10.1007/s10902-010-9209-7.

Augusto Landa, J. M., Aguilar Luzón, M. C. y Salguero de Ugarte, M. F. (2008). El papel de la IEP y del optimismo/pesimismo disposicional en la resolución de problemas sociales: Un estudio con alumnos de Trabajo Social. Revista Electrónica de Investigación Psicoeducativa, 6, 363-382.

Baker, F. (2001). The basics of item response theory. University of Maryland: College Park: ERIC Clearinghouse on Assessment and Evaluation.

Bandeira, M., Bekou, V., Silva Lott, K., Teixeira, M. y Silva Rocha, S. (2002). Validação transcultural do Teste de Orientação da Vida (TOVR). Estudos de Psicologia, 7, 251-258.

Brown, G. F. (1999). Principios de la medición en psicología y educación. México: Manual Moderno.

Browne, M. W. y Cudeck, R. (1993). Alternative ways of assessing model fit. In K. A. Bollen (Ed.), Testing structural equation models (pp. 136162). Long, JS: SAGE.

Carver, C. S. y Connor-Smith, J. (2010). Personality and Coping. Annual Review of Psychology, 61, 679-704. doi:10.1146/annurev. psych.093008.100352.

Carver, C. S., Scheier, M. F. y Segerstrom, S. C. (2010). Optimism. Clinical Psychology Review, 30, 879-889. doi:10.1016/j.cpr.2010.01.006.

Cliff, N. (1993). Dominance statistics: Ordinal Analyses to Answer Ordinal Questions. Psychological Bulletin, 114, 494-509. doi:10.1037/00332909.114.3.494.

Cramér, H. (1946). Mathematical Methods of Statistics. Princeton: Princeton University Press.

De Ayala, R. J. (2009). The theory and practice of item response theory. New York: Guilford Press.

Díaz Castela, M. M., Hale III, W. W., Muela, J. A., Espinosa-Fernández, L., Klimstra, T. y García-López, L. J. (2013). The Measurement of Perceived Emotional Intelligence for Spanish Adolescents with Social Anxiety Disorder Symptoms. Anales de Psicología, 29, 509-515. doi: http://dx.doi.org/10.6018/analesps.29.2.144271.

Downing, S. M. (2006). Twelve steps for effective test development. In S. M. Downing y T. M. Haladyna (Eds.), Handbook of test development (pp. 3-25). Mahwah, NJ: Lawrence Erlbaum Associates.

Eloranta, S., Arve, S., Lavonius, S., Routasalo, P., Lehtonen, A., Viitanen, M. e Isoaho, H. (2012). Positive life orientation in old age: A 15-year follow-up. Archives of Gerontology and Geriatrics, 55, 586-591. doi: 10.1016/j.archger.2012.04.010.

Elosua, P. y Zumbo, B. D. (2008). Reliability coefficients for ordinal response scales. Psicothema, 20, 896-901.

Extremera, N., Durán, A. y Rey, L. (2007). Perceived emotional intelligence and dispositional optimism-pessimism: Analyzing their role in predicting psychological adjustment among adolescents. Personality and Individual Differences, 42, 1069-1079. doi:10.1016/j.paid.2006.09.014.

Ey, S., Hadley, W., Allen, D. N., Palmer, S., Klosky, J., Deptula, D.,... Cohen, R. (2005). A new measure of children's optimism and pessimism: the youth life orientation test. Journal of Child Psychology and Psychiatry, 46, 548-558. doi:10.1111/j.1469-7610.2004.00372.x. 
Fernández-Berrocal, P., Extremera, N. y Ramos, N. (2004). Validity and reliability of the Spanish modified version of the Trait Meta-Mood Scale. Psychological reports, 94, 751-755.

Ferrando, P. J., Chico, E. y Tous, J. M. (2002). Propiedades psicométricas del test de optimismo Life Orientation Test. Psicothema, 14, 673-678.

Fischer, R. y Chalmers, A. (2008). Is optimism universal? A meta-analytical investigation of optimism levels across 22 nations. Personality and Individual Differences, 45, 378-382. doi:10.1016/j.paid.2008.05.008.

Flora, D. B. y Curran, P. J. (2004). An Empirical Evaluation of Alternative Methods of Estimation for Confirmatory Factor Analysis With Ordinal Data. Psychological Methods, 9, 466-491.

Gander, F., Proyer, R. T., Ruch, W. y Wyss, T. (2013). Strength-Based Positive Interventions: Further Evidence for Their Potential in Enhancing Well-Being and Alleviating Depression. Journal of Happiness Studies, 14, 1241-1259. doi:10.1007/s10902-012-9380-0.

Glaesmer, H., Rief, W., Martin, A., Mewes, R., Braehler, E., Zenger, M. e Hinz, A. (2012). Psychometric properties and population-based norms of the Life Orientation Test Revised (LOT-R). British journal of health psychology, 17, 432-445. doi:10.1111/j.2044-8287.2011.02046.x.

Gómez-Acosta, C. y Londoño, C. (2013). Predictive model of responsible comsuption of alcochol and typically not violent behaviour in adolescents Health and Addictions, 13, 23-34.

Gómez-Benito, J., Hidalgo, M. D. y Zumbo, B. D. (2013). Effectiveness of Combining Statistical Tests and Effect Sizes When Using Logistic Discriminant Function Regression to Detect Differential Item Functioning for Polytomous Items. Educational and Psychological Measurement, 73, 875-897. doi:10.1177/0013164413492419.

Hambleton, R. K., Swaminathan, H. y Rogers, H.J. (1991). Fundamentals of Item Response Theory. Newbury Park, CA: Sage.

Jovanovic, V. y Gavrilov-Jerkovic, V. (2013). Dimensionality and Validity of the Serbian Version of the Life Orientation Test-Revised in a Sample of Youths. Journal of Happiness Studies, 14, 771-782. doi:10.1007/ s10902-012-9354-2.

Kam, C. y Meyer, J. P. (2012). Do optimism and pessimism have different relationships with personality dimensions? A re-examination. Personality and Individual Differences, 52, 123-127. doi:10.1016/j. paid.2011.09.011.

Kline, R. B. (2011). Principles and Practice of Structural Equation Modeling. New York: Guilford Press.

Laranjeira, C. A. (2008). Portuguese Translation and Validation of the Revised Life Orientation Test (LOT-R). Universitas Psychologica, 7, 469-476.

Lemola, S., Raikkonen, K., Matthews, K.A., Scheier, M. F., Heinonen, K., Pesonen, A.K., . . . Lahti, J. (2010). A New Measure for Dispositional Optimism and Pessimism in Young Children. European Journal of Personality, 24, 71-84. doi:10.1002/per.742.

Londoño, C. (2009). Optimismo y salud positiva como predictores de la adaptación a la vida universitaria. Acta Colombiana de Psicología, 12, 95-107.

Londoño, C., Hernández, L. M., Alejo, I. E. y Pulido, D. (2013). Diseño y validación de la Escala de Optimismo Disposicional/Pesimismo-EOP. Universitas Psychologica, 12, 139-155.

López Puga, J. y García García, J. (2011). Dispositional optimism, pessimism and realism in technological potential entrepreneurs. Psicothema, 23, 611-616.

Lozano, L. M., García-Cueto, E. y Muñiz, J. (2008). Effect of the number of response categories on the reliability and validity of rating scales. Methodology, 4, 73-79. doi:10.1027/1614-2241.4.2.73.

Lyrakos, G. N., Damigos, D., Mavreas, V., Georgia, K. y Dimoliatis, I. D. K. (2010). A Translation and Validation Study of the Life Orientation Test Revised in the Greek Speaking Population of Nurses among Three Hospitals in Athens and Ioannina. Social Indicators Research, 95, 129142. doi:10.1007/s11205-009-9453-6.

Macbeth, G., Razumiejczyk, E. y Daniel Ledesma, R. (2011). Cliff's Delta Calculator: A non-parametric effect size program for two groups of observations. Universitas Psychologica, 10, 545-555.
Monzani, D., Steca, P. y Greco, A. (2014). Brief report: Assessing dispositional optimism in adolescence - Factor structure and concurrent validity of the Life Orientation Test-Revised. Journal of Adolescence, 37, 97-101. doi:10.1016/j.adolescence.2013.11.006.

Muñiz, J., Fidalgo, A. M., García-Cueto, E., Martínez, R. y Moreno, R. (2005). Análisis de los items. Madrid: La Muralla.

Nakano, K. (2004). Psychometric properties of the Life Orientation Test-Revised in samples of Japanese students. Psychological reports, 94, 849-855.

Park, N., Peterson, C. y Sun, J.K. (2013). Positive Psychology: Research and practice. Terapia Psicológica, 31, 11-19.

Pasch, K. E., Nelson, M. C., Lytle, L. A., Moe, S. G. y Perry, C. L. (2008). Adoption of risk-related factors through early adolescence: Associations with weight status and implications for causal mechanisms. Journal of Adolescent Health, 43, 387-393.

Pérez-Álvarez, M. (2013). La psicología positiva y sus amigos: en evidencia. Papeles del Psicólogo, 34, 208-226.

Peterson, C. (2000). The future of optimism. American Psychologist, 55, 44-55. doi:10.1037//0003-066x.55.1.44.

Peterson, C. y Seligman, M. E. P. (1984). Causal explanations as a risk factor for depression: Theory and evidence. Psychological Review, 91,347-374.

Plagnol, A. C. y Easterlin, R. A. (2008). Aspirations, Attainments, and Satisfaction: Life Cycle Differences Between American Women and Men. Journal of Happiness Studies, 9, 601-619. doi:10.1007/s10902008-9106-5.

Puskar, K. R., Bernardo, L. M., Ren, D., Haley, T. M., Tark, K. H., Switala, J. A. y Siemon, L. (2010). Self-esteem and optimism in rural youth: Gender differences. Contemporary Nurse, 34, 190-198.

Rauch, W. A., Schweizer, K. y Moosbrugger, H. (2007). Method effects due to social desirability as a parsimonious explanation of the deviation from unidimensionality in LOT-R scores. Personality and Individual Differences, 42, 1597-1607. doi:10.1016/j.paid.2006.10.035.

Rauch, W. A., Schweizer, K. y Moosbrugger, H. (2008). An IRT analysis of the personal optimism scale. European Journal of Psychological Assessment, 24, 49-56. doi:10.1027/1015-5759.24.1.49.

Salguero, J. M., Fernández-Berrocal, P., Balluerka, N. y Aritzeta, A. (2010). Measuring Perceived Emotional Intelligence in the Adolescent Population: Psychometric Properties of the Trait Meta-Mood Scale. Social Behavior and Personality: an international journal, 38, 1197-1209.

Samejima, F. (1969). Estimation of latent ability using a response pattern of grade scores. Psychometrika, 34 (4P2).

Scheier, M. F. y Carver, C.S. (1985). Optimism, coping, and helth: Assesment and implications of generalized outcome expectancies. Health Psychology, 4, 219-247.

Scheier, M. F., Carver, C. S. y Bridges, M. W. (1994). Distinguishing optimism from neuroticism (and trait anxiety, self-mastery, and self-esteem): A reevaluation of the life orientation test. Journal of Personality and Social Psychology, 67, 1063-1078. doi:10.1037/0022-3514.67.6.1063.

Schmitt, A., Gielnik, M. M., Zacher, H. y Klemann, D.K. (2013). The motivational benefits of specific versus general optimism. Journal of Positive Psychology, 8, 425-434. doi:10.1080/17439760.2013.820338.

Schweizer, K. y Koch, W. (2001). The assessment of components of optimism by POSO-E. Personality and Individual Differences, 31, 563-574. doi:10.1016/s0191-8869(00)00161-6.

Segerstrom, S. C., Evans, D. R. y Eisenlohr-Moul, T. A. (2011). Optimism and pessimism dimensions in the Life Orientation Test-Revised: Method and meaning. Journal of Research in Personality, 45, 126-129. doi:10.1016/j.jrp.2010.11.007.

Seligman, M. E. P. (2003). La auténtica felicidad. Barcelona: Vergara.

Seligman, M. E. P. (2008). Positive health. Applied Psychology: an International Review, 57, 3-18. doi:10.1111/j.1464-0597.2008.00351.x.

Sharpe, J. P., Martin, N. R. y Roth, K. A. (2011). Optimism and the Big Five factors of personality: Beyond Neuroticism and Extraversion. Personality and Individual Differences, 51, 946-951. doi:10.1016/j. paid.2011.07.033. 
Sireci, S. G. y Faulkner-Bond, M. (2014). Validity evidences based on test content. Psicothema, 26, 100-107. doi:10.7334/psicothema2013.256.

Snyder, C.R., Rand, K. y Sigmon, D. R. (2005). Hope theory: A member of the positive psychology family. In S. C.R. y L. S.J. (Eds.), Handbook of positive psychology (pp. 257-276). New York: Oxford.

Stipek, D. J., Lamb, M. E. y Zigler, E. F. (1981). OPTI: A measure of children's optimism. Educational and Psychological Measurement, 41, 131-143.

Sulkers, E., Fleer, J., Brinksma, A., Roodbol, P.F., Kamps, W. A., Tissing, W. J. E. y Sanderman, R. (2013). Dispositional optimism in adolescents with cancer: differential associations of optimism and pessimism with positive and negative aspects of well-being. British journal of health psychology, 18, 474-489. doi:10.1111/j.2044-8287.2012.02096.x.

Timmerman, M. E. y Lorenzo-Seva, U. (2011). Dimensionality Assessment of Ordered Polytomous Items With Parallel Analysis. Psychological Methods, 16, 209-220. doi:10.1037/a0023353.

Tusaie, K. R. y Patterson, K. (2006). Relationships among trait situational and comparative optimism: Clarifying concepts for a theoretically consistent and evidence-based intervention to maximize resilience. Archives of Psychiatric Nursing, 20, 144-150.

Vera-Villarroel, P., Córdova-Rubio, N. y Celis-Atenas, K. (2009a). Optimism Evaluation: Preliminary Analysis of the Life Orientation Test Revised Version (Lot-R) in Chilean Population. Universitas Psychologica, 8, 61-67.

Vera-Villarroel, P., Córdova-Rubio, N. y Celis-Atenas, K. (2009b). Optimismo versus Autoestima: Implicancia para la psicología clínica y la psicoterapia. Revista Argentina de Clínica Psicológica, 18, 21-30.

Vera-Villarroel, P., Pavez, P. y Silva, J. (2012). El rol predisponente del optimismo: hacia un modelo etiológico del bienestar. Terapia Psicológica, 30, 77-84.

Vigil-Colet, A., Morales-Vives, F., Camps, E., Tous, J. y Lorenzo-Seva, U. (2013). Development and validation of the Overall Personality Assessment Scale (OPERAS). Psicothema, 25, 100-106. doi:10.7334/ psicothema2011.411.

Xanthopoulou, D., Bakker, A. B. y Fischbach, A. (2013). Work Engagement Among Employees Facing Emotional Demands The Role of Personal Resources. Journal of Personnel Psychology, 12, 74-84. doi:10.1027/1866-5888/a000085.

Zenger, M., Finck, C., Zanon, C., Jiménez, W., Singer, S. e Hinz, A. (2013). Evaluation of the Latin American version of the Life Orientation TestRevised. International Journal of Clinical and Health Psychology, 13, 243-252.

Zumbo, B. D. (1999). A Handbook on the Theory and Methods of Differential Item Functioning (DIF): Logistic Regression Modeling as a Unitary Framework for Binary and Likert-Type (Ordinal) Item Scores. Ottawa, ON: Directorate of Human Resources Research and Evaluation, Department of National Defense. 